\title{
Reasons of Diagnostic Errors and the Ways to Overcome among Medical Residents
}

Shafaq Sultana, Farhat Fatima

\begin{abstract}
:
Arriving at an accurate diagnosis is one of the competencies prime of the medical practitioner. Errors may occur in the diagnostic process anywhere from the point of patient's initial assessment and interpretation of diagnostic tests, and even during follow-up and patient referral. Patient safety is gaining global precedence and in this context diagnostic errors are speculate as an important cause of harm to the patients. ${ }^{1}$ An awareness of the possible underlying factors leading to diagnostic errors, along with a repertoire of strategies to improve can be of great help to both junior and senior medical residents.
\end{abstract}

\section{INTRODUCTION:}

The burden of diagnostic errors is significant to the point that approximately $5 \%$ of adults who attended outpatient clinics endured diagnostic errors on an annual basis. Above $50 \%$ of these errors had detrimental consequences. This data reflects situation in a developed country and higher percentage of diagnostic errors is expected in developing countries, as access to resources and specialists is further limited there. ${ }^{1}$ Considering this explicit attempts by residents to improve their diagnostic accuracy can in the end increase Patient Safety standards, more so in a country where resources are scarce.

\section{Reasons for diagnostic errors:}

Possible causes for a resident making diagnostic errors could be:

He is committing a "no-fault error" because the patient presentation was either silent, atypical or mimicked a very common disease. They arise because of necessary short comings and the probabilistic nature of choosing a diagnosis on part of the physician. ${ }^{2}$

His errors could be a result of system errors which are due to inadequacy of the health care system. ${ }^{2}$

He could be making "cognitive errors", a critical subset of diagnostic errors, collectively referred as cognitive dispositions to respond (CDRs). These are especially

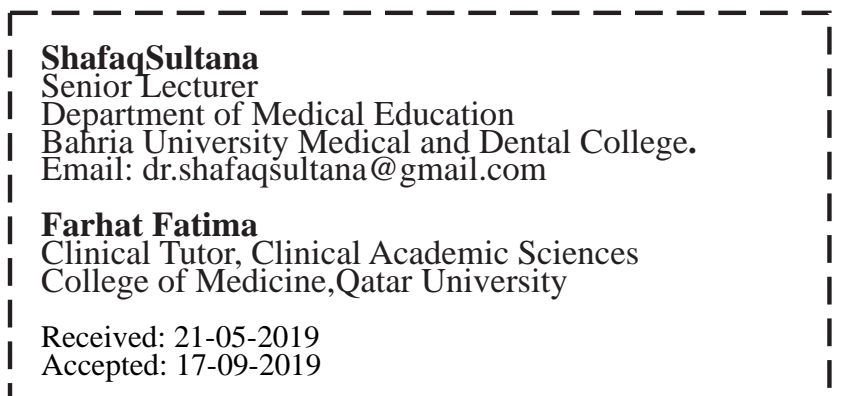

associated with failures in perception, failed heuristics and biases ${ }^{2-4,10}$ Studies have found processing biases like availability, base rate neglect, representativeness, confirmation bias, premature closure and many morea far more common cause of diagnostic error than knowledge gaps. ${ }^{3,4}$

However, according to psychological literature heuristics and rules of thumb are efficient mental strategies which may help clinicians cope with uncertain situations and overcome the limitations of time and data.They serve as powerful tools to cope up with the diagnostic challenges and usually lead to accurate decisions, though at the cost of predictable error reflecting the inherent biases associated with them. ${ }^{2-6,10}$

Diagnostic errors can also be a result of poor attitude towards best practices and over confidence.Studies on doctors self-assessment and error recall have found doctors to over rate themselves and they also had difficulty in recalling errors they made.,

\section{How to reduce Diagnostic errors:}

Following strategies can be employed by medical residents to reduce diagnostic errors:

Develop awareness by providing detailed and thorough characterization of known cognitive biases. Provide multiple clinical examples on the constructive as well as detrimental effects of cognitive biases on the diagnostic process. This will help them reflect on the specific effects of heuristics on clinical decisions. ${ }^{4,8,9,18}$

Train them to employ a reflective approach to problem solving. Studies have shown that if problem solving training is coupled with metacognitive training or other kinds of instruction, such as questioning it further enhances the problem solving abilities of the student. Only a self-directed, strategic, reflective learner can become a flexible, effective problem solver., ${ }^{4,9,13-15}$

Develop generic and specific cognitive forcing strategies to avoid predictable bias in particular clinical situations. $^{4,11}$

Provide them with adequate time for quality decision making. ${ }^{4}$

Provide them with timely and effective feedback so that errors are confronted early on and corrected. ${ }^{4,9,12,13}$

Promote residents to adopt a systematic approach to common problems. ${ }^{13}$

Promote them to practice worst case scenario medicine where in the worst possible diagnosis is considered 
first. This technique might lead to unneeded lab testing. To avoid this teacher should emphasize that it is not always necessary that if a diagnosis is considered it should be tested as well.The positive side of this approach is that serious diseases will be considered and evaluated..$^{9,10}$

Promote the use of why questions to reinforce the importance of examining all cases in detail. ${ }^{9}$

Teach and reinforce the value of clinical exam as it helps reduce diagnostic errors occurring as a result of premature closure and unpacking error. ${ }^{9}$

Promotingresidents to use diagnostic timeout effectively by making every effort not to frame the data using the current working diagnosis, can prove useful in preventing propagation of a diagnostic error. ${ }^{9}$ Learning strategies to foster elaborated and compiled knowledge should be employed for medical training. ${ }^{16}$

Acquisition of expert knowledge should be highly facilitated, as studies have shown that content is what derives the medical diagnostic work. ${ }^{8,16}$ Itshould be emphasized that nothing can substitute for knowledge attainment and clinical experience. ${ }^{16}$

Provide them with an understanding of the basic processes of problem solving because an awareness of general problem solving strategies can turn a person into a better problem solver. ${ }^{8,13}$

Promote them to focus on discovering and identifying the problem. Adequate time should be spent on this stage because the better a problem is conceptualized the better it is solved,particularly when coupled with high creativity and motivation on part of the solver., ${ }^{9,13,14}$ Promote them to use external representations of the problem whenever possible for example written or graphical. This is necessary because most problems encountered by physicians exceed the limited stores of sensory and short-term memory. This causes an inability to hold all relevant pieces of a problem in working memory. ${ }^{13,14}$

Diagnoses can be arrived at more precisely by engaging in more than one form of reasoning. Such an approach aids in maintaining balance between the analytical and non-analytical forms of reasoning particularly while tackling diagnostic challenges. ${ }^{19}$

\section{CONCLUSION:}

An awareness of the possible underlying factors leading to diagnostic errors, along with a repertoire of strategies to improve can be of great help to both junior and senior medical residents. They will have metacognitive awareness of their own practices while making diagnosis and can guide their juniors along similar lines.

\section{REFERENCES:}

1. World Health organization. Diagnostic errors: Technical series on safer primary care. c2016 [cited 2019 Feb24]. Available from https://apps.who.int/iris/bitstream /handle/10665/252410 /9789241511636eng.pdf;jsessionid=596169EC8715CFA42 14ABAACDEE5234C ? sequence $=1$

2. Mark G, Ruthanna G, Nancy F. Reducing diagnostic errors in medicine: what's the goal? Acad Med 2002;77(10):981-992.

3. Norman GR, Eva KW.Diagnostic error and clinical reasoning.Med Educ 2010;44:94-100.

4. CroskerryP.The importance of cognitive errors in diagnosis and strategies to minimize them. Acad Med 2003;78(8):775780 .

5. Elstein AS. Heuristics and biases: selected errors in clinical reasoning. Acad Med 1999;74:791-794.

6. Eva KW, Norman GR. Heuristics and biases: a biased perspective on clinical reasoning. Med Educ 2005;39:870872.

7. Berner ES, Graber ML. Over confidence as a cause of diagnostic error in medicine. Am J Med 2008;121(suppl):233.

8. Collard A, GelaesS, Vanbelle S et al. Reasoning versus knowledge retention and ascertainment throughout a problembased learning curriculum. Med Educ 2009;43:854-865.

9. Trowbridge RL.Twelve tips for teaching avoidance of diagnostic errors.MedTeach2008;30:496-500.

10. Croskerry P. Achieving quality in clinical decision making: cognitive strategies and detection of bias. AcadEmerg Med 2002;9(11):1184-1204.

11. Croskerry P. Cognitive forcing strategies in clinical decision making. Ann Emerg Med 2003;41:110-120.

12. Croskerry P. The feedback sanction. AcadEmerg Med 2000;7:1232-1238.

13. BruningRH,SchrawGJ,NorbyMM.Problem solving and critical thinking.In:CognitivePsychology and instruction.5th ed.Boston:Pearson Education Inc;2011:p. 160-191.

14. Bruning RH, Schraw GJ,NorbyMM.Introduction to cognitive psychology. In:CognitivePsychology and instruction. Boston: Pearson Education Inc; 2011;(5):1-12.

15. Epstein RM. Reflection, perception and the acquisition of wisdom. Med Educ 2008;42:1048-1050.

16. Fuks A, Boudreau JD, Cassell EJ. Teaching clinical thinking to first year medical students. Med Teach 2009;31:105-111.

17. Bruin ABH, Schmidt HG, RikersRMJP.The role of basic science knowledge and clinical knowledge in diagnostic reasoning:A structural equation modeling approach.Acad Med 2005;80(8):765-773.

18. Kassirer JP. Teaching clinical reasoning: case based and coached. Acad Med 2010;85(7):1118-1124.

19. Eva KW, Hatala RM, LeBlanc VR, Brooks LR. Teaching from the clinical reasoning literature: combined reasoning strategies help novice diagnosticians overcome misleading information. Med Educ2007;41:1152-1158.

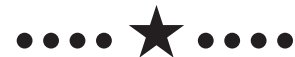

